Société d'histoire de la révolution de 1848 et des

révolutions du XIXe siècle

$52 \mid 2016$

Chrononymes. Dénommer le siècle

\title{
Les Hungry Forties
}

The Hungry Forties

The Hungry Forties

Anthony Howe

\section{Q OpenEdition}

\section{Journals}

Édition électronique

URL : http://journals.openedition.org/rh19/4989

DOI : $10.4000 /$ rh 19.4989

ISSN : $1777-5329$

Éditeur

La Société de 1848

\section{Édition imprimée}

Date de publication : 1 juin 2016

Pagination : 47-64

ISSN : $1265-1354$

\section{Référence électronique}

Anthony Howe, "Les Hungry Forties », Revue d'histoire du XIXe siècle [En ligne], 52 | 2016, mis en ligne le 01 juin 2019, consulté le 03 mai 2019. URL : http://journals.openedition.org/rh19/4989 ; DOI :

$10.4000 /$ rh19.4989 


\section{ANTHONY HOWE Les Hungry Forties*}

Loin d'être utilisé par les contemporains, le chrononyme The Hungry Forties (littéralement "les années 1840 affamées») fut inventé en 1903, une soixantaine d'années après la décennie qu'il qualifie. L'expression devint un élément de propagande essentiel à l'époque édouardienne, dans le cadre de la lutte politique entre le libre-échange (après l'abolition des Corn laws en 1846) et la réforme douanière, tentative menée par Joseph Chamberlain pour réintroduire des taxes protectrices sur les biens entrant en Grande-Bretagne. Dans ce contexte, l'expression de Hungry Forties cherchait spécifiquement à rappeler les années 1840 comme période de faim et de détresse dont les Britanniques avaient été libérés par l'abrogation des Corn laws en 1846. Ce combat avait été mené par le radical Richard Cobden; de manière significative, 1904 marqua non seulement le centenaire largement fêté de sa naissance, mais aussi la diffusion, sinon l'invention de la formule The Hungry Forties par la propre fille de Cobden, la militante libérale et éminente suffragiste Jane Cobden Unwin ${ }^{1}$. Début novembre 1904, en étroite collaboration avec son mari, le célèbre éditeur progressiste Thomas Fisher Unwin, Jane Cobden Unwin publia The Hungry Forties : Life under the Bread Tax : Descriptive Letters and Other Testimonies from Contemporary Witnesses [Les Hungry Forties. La vie à l'époque de l'impôt sur le pain. Lettres descriptives et autres témoignages de témoins contemporains], volume fondé sur les souvenirs de nombreuses personnes qui avaient vécu ce qu'elles se rappelaient comme une période de famine et de pauvreté. Alors que la Grande-Bretagne fut à nouveau, après 1903, le théâtre d'une bataille électorale autour du libre-échange, cet ouvrage devint aussitôt un best-seller et connut de nombreuses rééditions avant la Première Guerre mondiale. Par conséquent, l'expression Hungry Forties s'inscrivit profondément dans la mémoire politique et sociale, à telle enseigne

* Traduit de l'anglais par Laurent Bury.

1. «Ce titre fut inventé par la fille de Cobden, et la formule, utilisée chaque jour sur les tribunes politiques et par la presse, fait désormais partie du lexique littéraire fabriqué par la langue anglaise», Francis W. Soutter, Fights for Freedom: the Story of my Life, Londres, T. Fisher Unwin, 1925, p. 33. 
qu'elle était employée à la fin du $\mathrm{XX}^{\mathrm{e}}$ siècle, en plein linguistic turn, comme si elle avait été conçue à l'époque qu'elle désigne ${ }^{2}$.

L'invention et le succès de ce chrononyme inspirent donc aux historiens un certain nombre de questions. Pour certains, le problème premier est simplement la réalité matérielle que recouvre cette expression : "Avait-on si faim que cela dans les années 1840 ?" Les historiens débattent depuis longtemps de l'étendue de la pauvreté et des liens entre faim et politique populaire dans les années 1840 . Le premier historien à avoir analysé précisément les Hungry Forties, William H. Chaloner en 1957, en souligna l'inexactitude : la faim fut bien plus grave entre 1837 et 1842 que durant la période de reprise économique, à partir de $1843^{3}$; plus récemment, Frank Trentmann était du même avis ${ }^{4}$. Un peu à l'encontre de ce consensus actuel, Peter Gurney a voulu revenir sur "la centralité politique du débat sur la faim et les questions de consommation en général à cette époque», cherchant à rouvrir le débat sur les Hungry Forties, en envisageant comment le mouvement chartiste, autant que la Ligue pour l'abolition des lois sur les grains, "prenaient sens par rapport à la faim et se mobilisaient autour de la consommation $"^{5}$. L'Irlande, partie du Royaume-Uni qui connut une faim intense, durable et répandue pendant la "Grande Famine», n’était guère présente dans le livre de Jane Cobden Unwin, pas plus que la famine irlandaise ne joua de rôle significatif en métropole dans le débat sur les Hungry Forties ${ }^{6}$. En fait, le souvenir de la famine en Irlande élabora son propre lexique, sa propre mythologie et son historiographie, avec une trajectoire politique et culturelle bien différente, à l'intérieur d'un cadre nationaliste ${ }^{7}$. Dans les années 1960, alors que les Hungry Forties anglaises tendaient à disparaître du souvenir et du discours, quand les médias employaient parfois le terme, c'était en général dans le contexte du souvenir culturel de la famine irlandaise réinventé a posteriori ${ }^{8}$. Même

2. James Vernon note ainsi que le terme s'inscrivit dans «l'inconscient politique», et «les historiens et les romanciers reproduisent désormais cette formule comme une description des années 1840 par l'époque elle-même", Hunger. A Modern History, Londres, Belnap Press of Harvard University Press, 2007, p. 256.

3. William H. Chaloner, Hungry Forties, Londres, Historical Association, 1957.

4. «Les années 1840 ne furent pas pires que les décennies précédentes, ni même que les années 1850 et 1860», Frank Trentmann, Free Trade Nation, Oxford, Oxford University Press, 2008, p. 40.

5. Peter Gurney, 'Rejoicing in Potatoes: The Politics of Consumption in England during the Hungry Forties', Past \& Present 203 (mai 2009), p. 99-136, en particulier p. 101.

6. Jane Cobden Unwin, The Hungry Forties: Life under the Bread Tax : Descriptive Letters and Other Testimonies from Contemporary Witnesses, Londres, T. Fisher Unwin, 1904, p. 242-246.

7. On parlait en Irlande de «la Grande Faim» ou de la «Mauvaise Époque» pour évoquer la famine des années 1840. Cf. entre autres, Andrew Merry [pseudonyme de Mildred Darby], The Hunger: Being Realities of the Famine years: Ireland, 1845-1848, Londres, Andrew Melrose, 1910; Cathal Póirtéir, Famine Echoes, Dublin, Gill \&Macmillan, 1995, p. 280; Niall Ó Ciosáin, 'Famine memory and the popular representation of scarcity', in Ian McBride (ed.), History and memory in Modern Ireland, Cambridge, Cambridge University Press, 2001, p. 95-117; Peter Gray, 'Memory and the commemoration of the Great Irish Famine', in Peter Gray et Oliver Kendrick (eds), The memory of catastrophe, Manchester, Manchester University Press, 2004, p. 46-64.

8. Pour un usage récent à propos de l'Irlande, $c f$. Charlotte Boyce, 'Representing the Hungry Forties in image and verse: the Politics of Hunger in early Victorian periodicals', Victorian Literature and Culture 40, 2012, p. 421-449. 
par la suite, quand les historiens explorèrent l'arrière-plan des révolutions de 1848 en Europe, et devinrent plus conscients de la pénurie alimentaire sur l'ensemble du continent, l'expression prit une extension géographique plus large, bien que plus éloignée de son sens initial? .

Si le milieu universitaire continue à parler des Hungry Forties, cela témoigne de la force évocatrice de cette formule, plus d'un siècle après son invention. Pour en comprendre tant la popularité que la longévité, nous pouvons diviser l'histoire de ce chrononyme en plusieurs étapes : genèse, diffusion, imprégnation culturelle et persistance politique, révision savante et transmutation. Cette analyse révélera comment la décennie de 1840 fut investie d'une profonde signification culturelle et sociale, et politisée de manière très efficace et durable, pour devenir une réalité allant de soi, une division "naturelle» du temps et de l'histoire, plutôt qu'une construction historique spécifique. L'objectif global sera de comprendre les conditions sociales, politiques et culturelles qui ont donné naissance à ce chrononyme et d'esquisser les contours de son usage et de sa diffusion par la suite.

\section{L'invention des Hungry Forties}

La première apparition de l'expression Hungry Forties figure dans une lettre adressée par Jane Cobden Unwin à une réunion devant avoir lieu à Manchester pour s'opposer à «l'impôt alimentaire», après que Joseph Chamberlain avait annoncé sa campagne pour réintroduire des taxes sur toutes sortes de denrées entrant en Grande-Bretagne ${ }^{10}$. La formule prit un contenu plus développé dans une lettre au Times émanant d'un Libéral influent (et ancien Premier ministre), lord Rosebery : «ceux qui se rappelaient les misères du protectionnisme ne devraient laisser passer aucune occasion de faire part de leur expérience à leurs compatriotes ${ }^{11}$. La notion fut reprise par Thomas Fisher Unwin en février 1904, lorsqu'il lança dans la presse un appel à témoignage, pour tous ceux qui disposaient de documents pertinents ou, du fait de leur «âge et expérience», de souvenirs personnels qu'il s'engageait à publier en un volume "dans l'intérêt du libre-échange " ${ }^{12}$. Dans une lettre largement diffusée, il suggérait que des «factures de commerçants et des comptabilités domestiques privées datant des "années de la faim" auraient aussi un intérêt et une utilité à des fins d'étude comparative ${ }^{13}$. En fait, pratiquement aucune

9. Cormac O Gráda, Richard Paping, et Eric Vanhaute (eds), When the Potato Failed: Causes and Effects of the Last European Subsistence Crisis, Turnhout, Brepols, 2007; Mike Rapport, 1848: Year of Revolutions, Londres, Little Brown, 2008, p. 36.

10. The Times, 25 juillet 1903 , p. 12 .

11. Daté du 12 janvier 1904, Times, 15 janvier 1904, p. 4 a.

12. Comme l'écrivit Rosebery à Fisher Unwin, «rien ne pourrait être plus efficace pour endiguer la révolution fiscale», 5 février 1904, documents Cobden, CP154, folio 1, West Sussex Record Office [dorénavant WSRO].

13. Ce texte fut publié dans The Daily News, The Times, The Westminster Gazette, Reynold's Newspaper, The Christian World et The Methodist Times, entre autres. 
documentation ou archive de ce genre ne fut ensuite employée dans le vigoureux déploiement de propagande autour des Hungry Forties lors du débat opposant libre-échange et réforme des tarifs douaniers; celui-ci fut plutôt dominé par des lettres fondées sur les souvenirs personnels de cette époque, recueillies et présentées par Jane Cobden Unwin. Ces "véritables documents humains, "brèves et simples annales des pauvres", parlent d'eux-mêmes et portent la preuve indélébile de leur sincérité ${ }^{14}$. Le choix de textes auquel elle avait procédé s'insérait dans le contexte de «l'Angleterre rurale» qu'elle connaissait pour résider dans la maison de son père à Dunford, dans le village de Heyshott (Sussex), et lui donnait un accès de première main à des souvenirs de la communauté remontant jusqu'aux années $1840^{15}$. Par ailleurs, Jane était prête à prouver "que l'Angleterre agricole préfère de meilleurs habits et une meilleure alimentation à tous les avantages imaginaires à tirer d'une politique de Représailles, de Préférences ou de Protection ${ }^{16}$. Dans cette veine, elle et son mari étaient prêts en octobre 1904 à publier leurs témoignages documentaires comme "antidote pratique et efficace à la campagne furieuse et rageuse de ceux qui voudraient par une loi ramener l'Angleterre au temps des Hungry Forties» ${ }^{17}$.

Le contenu des Hungry Forties était donc dominé par la mémoire populaire plutôt que par les documents d'archives, mais son efficacité didactique venait de ce que livre faisait fusionner toute une gamme de souvenirs locaux en un tout national, créant un récit populaire de faim et d'oppression. Malgré tout, le contenu de l'ouvrage était fragmentaire, organisé en six régions (Midlands, East Anglia, Sud-Est, Wessex, Nord et autres), précédé d'une introduction par Jane Cobden Unwin et conclu par un chapitre dû à «Brougham Villiers» (pseudonyme rappelant le monde politique des années 1840, derrière lequel se cachait l'auteur progressiste F. J. Shaw), qui tirait les leçons de "l'Angleterre des Lettres ${ }^{18}$. L'introduction s'appuyait en grande partie sur les entretiens réalisés par Jane avec des membres âgés de la population locale, car elle avait été déçue par le manque de réactions dans le Sussex à l'appel lancé dans la presse. Ces souvenirs confirmaient une vision de l'amélioration du sort du peuple depuis les années 1840, le temps de profonde

14. Jane Cobden Unwin, The Hungry Forties, op. cit., p. 20.

15. The Hungry Forties était dédié «Ä ceux qui, dans son village natal de Heyshott, ont connu et aimé Richard Cobden".

16. Ibidem, p. 18.

17. Ibidem, p. 54 ; Accord sur les droits d'auteur entre E. J. C. Unwin et T. F. Unwin, éditeurs, 28 octobre 1904, CP 1081, WSRO.

18. L'Irlande, le Pays de Galles et l'Écosse étaient moins bien représentés, Jane Cobden Unwin regrettant "le silence général de l'Ecosse», à l'exception d'un exposé "excessivement intéressant", venant d'Aberdeen, Hungry Forties, 227, 236-242. La lecture de la presse indique que l'expression était en usage courant en Écosse dans le cadre d'un débat britannique plus large, et non exclusivement focalisé sur la famine écossaise des années 1840. Pour l'Écosse, $c f$. Thomas M. Devine, The great Highland Famine: Hunger, Emigration and the Scottish Highlands in the nineteenth century, Edinburgh, John Donald, 1988; pour la presse, $c f$. entre autres Dundee Daily Press (14 janvier 1910, 24 août 1911, 20 janvier 1913); Falkirk Herald (29 janvier 1910, 31 décembre 1910). 
détresse et d'incendie des meules de foin dans les campagnes étant désormais bien loin ${ }^{19}$. Le contenu des autres chapitres était très variable mais l'un des chapitres les plus complets, celui consacré à l'East Anglia, se fondait surtout sur un recueil antérieur de souvenirs locaux, publié par un éminent Libéral de la région, Robert Winfrey, sous l'égide de la Free Trade Union, en $1903^{20}$. Ce livre avait servi de modèle à Jane Cobden Unwin, avec bon nombre d'entretiens accordés par des habitants âgés du Norfolk, des femmes pour la plupart, et avec l'immédiateté conférée par l'ajout de photographies des pauvres «méritants». Alors que le texte de Winfrey s'inscrivait dans le cadre de sa campagne locale pour rallier le suffrage des ouvriers (il allait remporter le siège du South-West Norfolk en 1906), Jane Cobden Unwin reprit les documents qu'il avait publiés dans le but d'incorporer la mémoire locale à une mémoire nationale, de manière tout à fait efficace et visuellement frappante. D'autres souvenirs locaux furent également inclus : par exemple, dans le chapitre "Wessex», le révérend W. D. Sargeant, de Stoke Abbott, dans le Dorset, racontait la vie d'un de ses locataires, tandis qu'une certaine Miss Benjafield, de Stalbridge, avait interrogé un grand nombre de personnes âgées et transcrit leurs souvenirs très présents. D'autres souvenirs étaient réellement individualistes, comme dans le cas de Lucy Buckland, la seule femme à prendre directement la parole : en 1904, elle avait 88 ans et habitait Westcliff-on-Sea, mais nous pouvons reconnaître en elle Lucy Blunt, modiste qui avait épousé en 1842 William Buckland, batteur d'or à Islington; en 1851, ils avaient trois enfants, avec une mère et une belle-mère à domicile, dans une maisonnée dont Lucy notait les dépenses dans le moindre détail ${ }^{21}$. De manière plus idiosyncrasique, on peut identifier "James Hawker de Padby» comme James Hawker, habitant Oadby dans le Leicestershire, qui se rendit célèbre en tant que braconnier. Né en 1836, il attribua toujours son braconnage à la faim subie dans sa jeunesse. Ses mémoires hauts en couleurs, écrits en 1903-1904, n'employaient pas l'expression "Hungry Forties», mais parlaient des «jours sombres du protectionnisme», et de la menace que la réforme de Chamberlain constituait pour les pauvres. Animé par une vigoureuse conscience de classe, Hawker montait volontiers sur les tribunes des libéraux pour s'exprimer dans cette veine ${ }^{22}$.

19. En fait, dans ce contexte, beaucoup rendaient hommage au rôle local de Richard Cobden comme employeur, plutôt qu'au héros national qui avait obtenu l'abrogation des Corn laws.

20. Free trade and Protection: which is it to be. Read this and see. 1 How Norfolk people suffered in the old days of Protection. 2. How Mr Chamberlain's Scheme would again tax your food, Free Trade Union, 1903, exemplaire conservé à la Millennium Library, Norwich. Je remercie Alun Howkins d'avoir identifié le tract utilisé par Jane Cobden-Unwin. Des souvenirs similaires étaient mobilisés dans le chapitre "In the days of protection", du recueil dirigé par Henry W. Massingham, Labour and Protection, Londres, T. Fisher Unwin, 1903, p. 118-142.

21. Jane Cobden Unwin, The Hungry Forties, op. cit., p. 136-137; données biographiques obtenues sur ancestry.co.uk.

22. Ibidem, p. 77-82; Garth Christian (ed.), James Hawker's Journal: A Victorian Poacher, Londres, Oxford University Press, 1961, en particulier. p. 15, 95. Ce livre fut dédié à John Tudor Walters, qui se trouvait être le fils d'un correspondant de Richard Cobden dans les années 1850, CP 4, WSRO. Hawkins avait une connaissance locale directe de l'un des Tariff Commissioners de 
À partir de ces souvenirs disparates et sans grande cohérence, mais vivants et authentiques, The Hungry Forties fut conçu comme un récit populaire où la faim n'était ni la conséquence naturelle du fonctionnement du marché, ni le sous-produit inévitable de la Révolution industrielle britannique, mais le résultat du "mauvais gouvernement», de choix politiques visant à servir les intérêts de propriétaires sans cœur et de fermiers intransigeants. C'était revenir à une économie morale antérieure, et l'abrogation des lois sur les grains était présentée comme le sauvetage quasi biblique du peuple arraché à l'oppression, "servitude égyptienne d'où il avait été délivré» ${ }^{23}$.

Autre point notable, comme l'affirmait Brougham Villiers, l'expression Hungry Forties en vint à résumer l'ensemble de la période allant de 1815 à 1846, c'est-à-dire du vote des Corn laws jusqu'à leur abolition. Nombre de textes écrits par la suite unifiaient ainsi ces décennies en une période de "malnutrition systématique de toute la nation" à cause de mauvaises décisions prises après les guerres napoléoniennes. Comme l'écrivait Brougham Villiers, «l'île, puisqu'elle n'était plus assiégée par ses ennemies, devait être assiégée par ses dirigeants. Les taxes douanières devaient annuler les méfaits de la paix et rendre coûteux les produits de la terre ${ }^{24}$. Dans ces conditions, les pauvres (terme quasi synonyme de «la nation») se mirent à boire du thé à base de croûtes brûlées, et à manger de grossiers ersatz de pain, des navets souvent dérobés la nuit dans les champs, "base alimentaire sur laquelle il semble impossible de maintenir une vie saine $»^{25}$. Cet usage du passé avait pourtant des intentions polémiques très présentes. Tout en brandissant le spectre d'un retour au passé, le tract mettait à plusieurs reprises l'accent sur le nouveau modèle de consommation de la population (qui incluait les raisins secs, le pain de froment, la viande, le sucre, le thé) et dénonçait très habilement la Réforme des tarifs comme un risque démoniaque pour la nourriture des pauvres ${ }^{26}$; les Hungry Forties n'étaient pas évoquées comme simple menace de retour à la famine, mais comme menace pour le niveau de vie meilleur dont jouissaient de nombreux consommateurs édouardiens.

\section{Politique de propagande dans l'ANgleterre Édouardienne}

La publication de The Hungry Forties s'avéra un coup de maittre en matière de propagande ${ }^{27}$. Néanmoins, l'impact du livre venait plus du contexte que

Chamberlain, John Corah, fabricant de bas à Leicester et propriétaire terrien, Journal, p. 95; $c f$. Andrew Marrison, British Business and Protection, 1903-1932, Oxford, Clarendon Press, 1996, p. 50, $61,180,185$.

23. Jane Cobden Unwin, The Hungry Forties, op. cit., p. 274.

24. Ibidem, citations p. 252 et 255.

25. Ibidem, p. 263.

26. Chamberlain lui-même devint l'incarnation de cette menace, $c f$. Jane Cobden Unwin, The Hungry Forties, op. cit., p. 62, 68-69 sq.

27. Anthony Howe, 'Towards the Hungry Forties: free trade in Britain, c. 1880-1906' in Eugenio 
de son contenu détaillé. Le titre seul signifiait la mobilisation efficace de la mémoire historique au service de la défense du libre-échange, tout en incarnant une vision de l'histoire déjà profondément enracinée dans la culture politique britannique. En effet, le mythe existait déjà à l'intérieur d'un "imaginaire démocratique» avant d'être exprimé en mots ${ }^{28}$. Cela s'accordait bien avec d'autres récits populaires devenus très répandus vers 1900 : par exemple, des ouvrages comme A Village Politician: The Life-Story of John Buckley (1897), de John Buckmaster, offraient un exposé vivant de la bataille contre les lois sur les grains par un de ses protagonistes les plus prolétariens; en 1903, When I was a Child, par "Un vieux potier" (Charles Shaw, 18321906), évoquait en détail les années 1840, dont les grèves dites Plug Plot de 1842 et les Corn laws, et était en partie écrit comme une mise en garde contre le retour du protectionnisme ${ }^{29}$. D'autres autobiographies ouvrières réitéraient le message - les années 1840 avaient été une décennie de détresse populaire et de mécontentement. En 1893, le leader des ouvriers agricoles, Joseph Arch, écrivait : «J'ai encore très clairement en tête les horreurs de cette époque, comme si elles avaient été marquées en moi avec un fer brûlant. Je ne saurais les oublier ${ }^{30}$. La menace d'un retour au protectionnisme déclencha aussi un raz-de-marée d'ouvrages chargés de réveiller le souvenir des années 1840, en particulier chez Fisher Unwin. On peut citer non seulement les éditions populaires, abrégées ou anniversaires du texte canonique de Morley, Life of Richard Cobden, mais des titres comme Labour and Protection (1903), mettant l'accent sur les bienfaits du libre-échange pour la classe ouvrière depuis 1846, et la réimpression des très appréciés Corn Law Rhymes, recueil de poèmes d'Ebenezer Elliott ${ }^{31}$. Tous ces écrits insistaient sur les avantages acquis par le peuple britannique depuis 1846, ce qui s'accordait bien avec la vision générale du passé qu'avaient les Victoriens et les Edouardiens : même sans recourir à l'expression Hungry Forties, il existait un sentiment de progrès par rapport à l'époque sombre allant de 1815 à 1846. Cette idée remontait aux premiers historiens du XIX siècle, comme Harriet Martineau avec son History of England during the Thirty Years Peace, 1816-1846 (1850) ${ }^{32}$. Ce récit était également inscrit au cour des manuels scolaires de la fin du siècle, qui proposaient une image de progrès, dès lors que le peuple avait été peu à peu

Biagini (ed.). Citizenship and Community: Liberals, radicals and collective identities in the British Isles, 1865-1931, Cambridge, Cambridge University Press, 1996, p. 193-218; Anthony Howe, Free Trade and Liberal England, 1846-1946, Oxford, Clarendon Press, 1997, p. 232, 259, 263, 271; Frank Trentmann, Free Trade Nation, op. cit., p. 34-45.

28. Selon Patrick Joyce, le mythe des Hungry Forties «allait devenir l'un des plus durables et des plus inspirants parmi les mythes politiques du siècle", in Democratic Subjects: the self and the social in nineteenth-century England, Cambridge, Cambridge University Press, 1994, p. 129-130.

29. Ce livre fut par la suite une des sources d'Arnold Bennett pour son roman Clayhanger (1910).

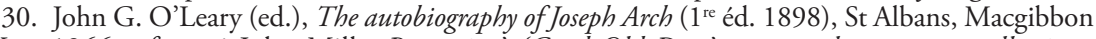
\& Kee, 1966; cf. aussi John Mills, Protection's 'Good Old Days': some early century recollections, Manchester, Sherratt \& Hughes, 1903.

31. Curieusement, Lord Rosebery fit la même suggestion à Unwin, $c f$. note 12.

32. Anthony Howe, Free Trade... op. cit., p. 31, 32. 
soulagé des lois oppressives ${ }^{33}$. Dans cette veine, une «histoire populaire» des Corn laws parue au début du XXe siècle, s'appuyait sur le présupposé que le but de cette législation "était de maintenir le cours du blé aussi près du prix de famine que pourraient le supporter la masse de la population $»^{34}$. Une semblable conception du progrès s'était répandue dans toute une gamme de dialogues culturels, d'autant plus présents dans l'Angleterre édouardienne que l'intérêt pour l'Histoire était en plein essor ${ }^{35}$.

Cette vision du passé, qui n'était pas un âge d'or mais «les jours sombres du protectionnisme», prit une force politique nouvelle avec la campagne du parti libéral pour défendre le libre-échange contre la menace croissante de la réforme des droits de douane. Les survivants des Hungry Forties, comme plus tard ceux de l'Holocauste, furent enrôlés par le parti et prirent régulièrement la parole sur ses tribunes, notamment James Hawker, Joseph Arch et celui qui se faisait appeler le "vieux chartiste», W. H. Chadwick ${ }^{36}$. Vivantes incarnations des Hungry Forties pour l'électorat populaire, ils conféraient immédiateté et résonance au message selon lequel la Grande-Bretagne risquait de retourner à la pauvreté de jadis. De plus, sur le plan politique, loin d'apparaître comme un concept périmé, la résurrection des Hungry Forties contribua à associer le parti libéral au fort courant de réforme agraire et de mesures progressistes contre la pauvreté rurale, tandis que dans les zones urbaines, les libéraux pouvaient se présenter comme les défenseurs du bien-être ouvrier contre les fausses promesses de Chamberlain. Parmi les représentations picturales des Hungry Forties, une des plus efficaces fut l'affiche de Robert Morley qui gagna le concours du National Liberal Club en 1905, avec cette légende : "Prêts à retourner en arrière? Rappelez-vous les Hungry Forties. La réforme douanière, ça veut dire des trusts pour les riches, des miettes [crusts] pour les pauvres $»^{37}$. L'image de la grosse miche et du petit pain, courante dans les années 1840, fut largement réutilisée dans les programmes politiques et dans les nouveaux médias culturels, avec le fol engouement pour les cartes postales représentant des miches de pain. Le motif des Hungry Forties s'inscrivait ainsi dans une vaste conceptualisation des années 1840 comme lutte entre riches et pauvres, privilégiés et industrieux, monopolistes et peuple, tyrans et opprimés, discours qui conservait toute sa puissance d'évocation

33. Valerie Chancellor, History for their Masters: Opinion in the English History Textbook, 18001914, Bath, Adam \& Dart, 1970; «le principe de l'Histoire était représenté par le Libre-Échange, celui de la réaction par le Protectionnisme», Patrick Joyce, Visions of the People: Industrial England and the question of class, 1848-1914, Cambridge, Cambridge University Press, 1991, p. 189.

34. Mary A. M. Marks, The Corn laws: A popular history, Londres, A. C. Fifield, 1908, p. 7. Ce livre contenait une dénonciation sévère des intérêts terriens du point de vue d'une avocate de la réforme mais mentionnait, contre toute attente, les «hungry twenties», les années 1820 , plutôt que les Hungry Forties.

35. Paul Readman, 'The Place of the Past in English Culture, c. 1890-1914', Past \& Present 186 (2005), p. $147-199$.

36. Hawkins, Journal, p. xvi; Joseph Arch, Manchester Guardian, 15 janvier 1906, p. 10; Newbould T. Palmer, In memoriam William Henry Chawick, the 'Old Chartist', Luton, 1908.

37. Frank Trentmann, Free Trade Nation, op. cit., planche IV entre les pages 210 et 211. 
dans l'Angleterre édouardienne ${ }^{38}$. De manière plus décisive encore, ce devint un message adressé au consommateur, à une époque où l'on s'intéressait de plus en plus au budget de la classe ouvrière dans les débats sur la pauvreté et dans les enquêtes sociales plus nombreuses. La Réforme douanière fut décrite comme une menace potentielle pour le budget soigneusement équilibré des foyers ouvriers, pour la vie de famille, pour ses rôles genrés et son économie morale ${ }^{39}$.

Il est impossible de mesurer quelle fut exactement l'efficacité de l'expression Hungry Forties dans la victoire électorale des libéraux en 1906, mais il ne semble guère faire de doute que la formule conquit sa valeur durable entre 1904 et $1906^{40}$. Avant même la parution de The Hungry Forties, un extrait des témoignages du Sussex avait été diffusé dans toutes les maisons de Horsham durant l'élection partielle de novembre $1904^{41}$. Après la première édition de 1904, une édition populaire fut publiée en 1905 (au prix de 6 pence), rééditée en 1909, une version abrégée en 1910 (réimprimée la même année), avec une édition complète à un penny, tirée à 110000 exemplaires en 1912, puis encore 100000 en fin d'année ${ }^{42}$. Il est également clair que les termes associés, comme "nourriture", "faim» et "pain» conquirent du terrain dans les discours électoraux ${ }^{43}$. Nous avons vu aussi que les libéraux mobilisèrent des défenseurs comme Arch et Hawker. Au parlement, les députés surent employer l'expression dans leurs débats; par exemple, sir John Leese, fils d'un militant anti-Corn Law, déplora la potentielle hausse des prix liée aux droits de douane qui, "après soixante ans d'abondance et de prix comparativement bas», "allait ranimer les tristes et lointains souvenirs de ce qu'on appelait les Hungry Forties» ${ }^{44}$. Sir John Lubbock, éminent banquier de la City, déplorait lui aussi un politique qui lui semblait «radicalement rétrograde et qui nous ramènerait aux temps terribles des Hungry Forties ${ }^{45}$. À la chambre des Lords, l'évêque radical de Hereford, John Percival, assez âgé pour se rappeler les Hungry Forties dans le nord de l'Angleterre, renchérit: «La misère atroce de cette époque est gravée dans ma mémoire [...] Je n’ai aucun désir de revoir quoi que ce soit de semblable ${ }^{46}$. Cette mémoire politisée fut propagée de mille manières, notamment dans la propagande des Free Trade Union et des Women's Free Trade Union, ainsi que d'autres organismes

38. Anthony Howe, Free Trade and Liberal England, op. cit., p. 271.

39. Frank Trentmann, Free Trade Nation, op. cit., p. 69-133. Pour une illustration, voir plus bas, p. 57.

40. Pour un usage récent, Robert Tombs, The English and their History: the first thirteen centuries, Londres, Allen Lane, 2014, p. 544.

41. Times, 5 novembre 1904, p. 12.

42. Chiffres tirés de l'édition de 1912; Frank Trentmann, Free Trade Nation, op. cit., p. 41.

43. Luke Blaxill, The Language of British Electoral Politics, 1880-1910, thèse de doctorat non publiée, King's College, Londres, 2012, en particulier le chapitre IV.

44. Hansard's Parliamentary Debates [dorénavant Hansard], 4e série (144), c. 394, 4 avril 1905; voir aussi Ibidem (185), c. 782, 4 mars 1908.

45. $\operatorname{Ibidem~(189),~c.~414,~} 21$ mai 1908.

46. Ibidem, $5^{\text {e }}$ série (4), c. 1083, 25 novembre 1909. 
du parti libéral, dont la Liberal League et la Women's Liberal Association ${ }^{47}$. Sans oublier une pièce de théâtre jouée à la Free Trade Union, intitulée $A$ message from the Forties: A Free Trade masque, datant d'environ 1909 (Trentmann a montré qu'elle avait été conçue par l'épouse de l'employeur progressiste Alfred Mond $)^{48}$. Paradoxalement aussi, les Hungry Forties étaient une formule à laquelle durent se colleter les partisans de la réforme douanière; en un sens, leurs démentis répétés mais inefficaces, selon lesquels les taxes n’annonçaient pas un retour aux années 1840, confirmaient que les avocats du libre-échange avaient imposé des termes du débat. Comme le dit Morrison Bell, "Chaque discussion sur la réforme douanière se réduit donc toujours à la petite et à la grosse miche de pain des Hungry Forties $»^{49}$. Fait significatif, lord Derby affirma, pour soutenir un changement de la politique unioniste en 1913, que cette mesure était nécessaire parce que les Hungry Forties s'étaient avérées un "argument très puissant en faveur de leurs [opposants] „ $5^{0}$. Autre signe de la force de cette formule, les partisans de la réforme douanière tentèrent de reprendre la main dans le débat, notamment en façonnant des arguments aptes à séduire les consommateurs autant que ceux des libre-échangistes ${ }^{51}$. Néanmoins, la riposte de la réforme douanière fut en soi la preuve supplémentaire de l'impact "énorme» qu'avaient eu les Hungry Forties sur le discours politique édouardien ${ }^{52}$.

Enfin, même si les Hungry Forties étaient surtout présentes dans le discours des libéraux ${ }^{53}$ et si l'analyse socialiste naissante se méfiait de nombreux éléments de l'économie politique libérale ${ }^{54}$, leur souvenir était largement soutenu par le mouvement ouvrier, non seulement parmi les ouvriers agricoles mais aussi dans les zones industrielles, surtout là où la mémoire des Hungry Forties était encore très vive, et associée à des événements comme les grèves dites «Plug Plot» de 1842 dans le Lancashire, point central de la tendance révolutionnaire du mouvement chartiste, qui coïncida avec le pire de la dépression dans le Nord industriel ${ }^{55}$. Au sein de cette tradition, Allen

47. WSRO, CP 154. fo. 63 brouillon de brochure, "The Hungry Forties" pour la Women" Free Trade Union, 25 février 1908; The bad days of protection (Liberal Publications Department, brochure 1993, 1904); They remember: the miseries of protection (Liberal League, $\left.\mathrm{n}^{\circ} 125,1904\right)$; Frank Trentmann, Free Trade Nation, op. cit., p. 41.

48. Ibidem, p. 34-35.

49. Hansard, 5 ${ }^{\mathrm{e}}$ série (22), c. 2342, 15 mars 1911.

50. Times, 20 janvier 1913 , p. 8.

51. Blaxill, 'Language', loc. cit., p. 202-207.

52. Frank Trentmann, Free Trade Nation, op. cit., p. 41.

53. Anthony Howe, 'Popular Political Economy' in David Craig et James Thompson (eds), Languages of Politics in ninetenth-century Britain, Basingstoke, Palgrave Macmillan, 2013, p. 119-141.

54. Frank Trentmann, 'Wealth versus Welfare: the British Left between Free Trade and national political economy before the First World War', Historical Research 70, 1997, p. 70-98.

55. Malcolm Chase, Le chartisme. Aux origines du mouvement ouvrier britannique (1838-1858), traduction L. Bury, Paris, Publications de la Sorbonne, 2013, p. 272-294. En 1932 encore, un inspecteur des usines dans le Lancashire se plaignait en ces termes : «Vous savez que les gens de Lancashire ont l'air incapables d'oublier les hungry forties, comme si ce souvenir avait été gravé en eux", mentionné par la femme politique travailliste Ellen Wilkinson, 'Born and Bred in Lancashire', The Listener 881, 29 novembre 1945, p. 617. 
Clarke, écrivain et éditeur socialiste extrêmement populaire, ayant une profonde connaissance de la mémoire populaire et des traditions dialectales du Lancashire, souvent perçu lui-même comme «la voix du peuple», publia en 1914 "The Men who fought for us" in the Hungry Forties : a tale of pioneers and beginnings ["Les hommes qui se sont battus pour nous" pendant les Hungry Forties : une histoire de pionniers et de commencements $]^{56}$. La morale du conte de Clarke était la victoire finale des coopératives (les «Equitables Pionniers", de Rochdale sont très présents dans le récit), mais l'épilogue énonçait la leçon historique : les troubles du passé «sont étroitement reflétés par les [...] hommes politiques de notre époque ${ }^{57}$. Autre point intéressant, à la manière de The Hungry Forties, Clarke en tirait des enseignements pour la participation des femmes aux questions politiques, «De la bonne nourriture à bon marché, des foyers heureux, tout ça est autant l'affaire d'une femme que d'un homme, et même davantage car le soin du foyer est le travail de la femme. La coopération n'est pas une question de sexe ${ }^{58}$. Plus généralement, le mouvement coopératif voyait la défense des consommateurs comme sa mission et faisait directement remonter ses origines aux Hungry Forties ${ }^{59}$. Au sein de cette mémoire institutionnelle, il devint courant de voir la naissance du mouvement coopératif comme le résultat (et en partie le remède) des Hungry Forties; l'histoire du mouvement publiée en 1944, volume appelé à faire autorité, s'ouvrait sur un chapitre consacré aux Hungry Forties, et la détresse de cette époque fut évoquée dans une émission de radio destinée aux États-Unis et dans un défilé populaire conçu pour célébrer le centenaire ${ }^{60}$. En 1959 encore, Albert Alexander (1885-1965), fils d'un forgeron, désormais comte Alexander of Hillsborough, membre de longue date du mouvement coopératif, avait l'habitude d'en expliquer la genèse comme une réaction à la "famine des Hungry Forties» ${ }^{61}$.

\section{IMPRÉgNATION CULTURELLE ET PERSISTANCE POLITIQUe après la Première Guerre mondiale}

La lutte entre libre-échange et protectionnisme, par le biais de laquelle la formule Hungry Forties s'était répandue dans toute la nation avant 1914, se transforma rapidement après la Première Guerre mondiale, même si elle

56. Manchester, Co-Operative Newspaper Society, 1914. Pour Clarke, cf. Dictionary of Labour Biography, V, Joyce M. Bellamy et John. Saville (eds), Basingstoke, Macmillan, 1979, p. 64-70; Patrick Joyce, Visions of the People, op. cit., p. 301-302.

57. Clarke, The Men who fought for us, op. cit., p. 279.

58. Ibidem, p. 269.

59. Cf. Tweedell, directeur d'une Co-operative Wholesale Society, cité dans The Times, 7 août, 1907, p. 10.

60. George D. H. Cole, A Century of Co-operation, 1844-1944, Londres, Co-operative Union, 1944, chapitre I, The Hungry Forties; Manchester Guardian, 22 décembre 1943, p. 6; Manchester Guardian, 20 juin 1944, p. 6.

61. Hansard, $5^{\mathrm{e}}$ série (217), c. 484, 30 juin. 
revint sur le mode mineur à plusieurs moments critiques, et surtout dans la crise politique de 1931-3262. De toute évidence, l'expression restait tout à fait identifiable pour le public instruit; elle fit son entrée dans l'Oxford English Dictionary en $1933^{63}$ et était régulièrement employée dans toutes sortes de contextes politiques mais aussi culturels et littéraires. Tout d'abord, elle restait un trope employé par nombre de personnalités littéraires de second plan. On songe par exemple au premier professeur de Littérature anglaise à l'université de Leeds, explorateur passionné du folklore et de la culture populaire, qui publia en 1918 un poème en dialecte intitulé "The Hungry Forties", qui présentait la réforme douanière comme un assaut contre "le manger des ouvriers", menaçant de "nous renvoyer à not' vieille misère " ${ }^{64}$. Mais la large acceptation culturelle des Hungry Forties influença la réception critique d'un roman dû à un écrivain bien plus connu de l'entre-deuxguerres, Sheila Kaye-Smith. À propos de Susan Spray, un critique écrivit : «Elle arrive à exprimer jusqu'aux maux d'estomac des Hungry Forties; quand elle décrit le budget d'un ouvrier agricole, ce n'est pas juste un document, mais un document humain ${ }^{65}$. En 1930 suivit l'ouvrage publié par la Société pour la diffusion de la connaissance chrétienne (Society for the Promotion of Christian Knowledge), Sons of Want: a story of the hungry forties, évoquant les Hungry Forties dans un récit exemplaire où la défaite du radicalisme politique (les grèves "Plug Plot» chartistes) conduit à la régénération religieuse sous la forme du socialisme chrétien. Son auteur, Lilian Howard Dalton, avait produit depuis 1913 nombre de récits exhortatifs, en rapport avec l'esclavage et l'activité missionnaire, mais aussi, en 1924, un recueil de League of Nations Stories. Également située dans le contexte des grèves de 1842, la pièce Doctor Scholefield: An incident of the Hungry Forties ${ }^{66}$, créée sur la scène du Manchester University Settlement en décembre 1934, était l'œuvre de Mary Danvers Stocks, future baronne Stocks, suffragiste connue et réformatrice de l'assistance sociale, qui avait étudié l'économie à la LSE avant la guerre ${ }^{67}$. Dans une veine un peu plus légère apparut en 1939 le personne de l'Oncle Silas, imaginé par le romancier populaire H.E. Bates (1905-1974) : ce charmant voyou local "né au début des Hungry Forties» dans le Northamptonshire s'inspirait de l'histoire d'un lointain ancêtre de l'auteur, Joseph Betts ${ }^{68}$. Bien que disparates, toutes ces références littéraires suggèrent que l'expression Hungry Forties resta identifiable entre les deux guerres, toujours apte à

62. Frank Trentmann, Free Trade Nation, op. cit., p. 189-190 sq.

63. W. H. Chaloner, Hungry Forties, op. cit., p. 6.

64. Frederick W. Moorman, Songs of the Ridings, Londres, Elkin Mathews, 1918, p. 49-50. Ce poème fut dédié à la section du Yorkshire de la Workers' Educational Association et fut d'abord publié dans la presse locale, peut-être bien auparavant, comme contribution au débat édouardien.

65. 'Present Day and the Hungry Forties', Manchester Guardian, 8 novembre 1928, p. 13.

66. Manchester, Sherratt \& Hughes, 1936.

67. Voir Mary D. Stocks, My Commonplace Book, p. 159-160, "un morceau d'histoire brute».

68. H.E. Bates, My Uncle Silas, Londres, Jonathan Cape, 1939, Préface, p. 9. Recueil de nouvelles, avec illustrations d'Edward Ardizzone; un deuxième recueil suivit en 1957, puis des adaptations très réussies pour le grand et le petit écran. 
connecter le public au souvenir d'un passé de plus en plus distant. Par ailleurs, cet appel à la mémoire était volontiers utilisé dans un nouveau média, la radio, qui proposa notamment un débat animé sur les droits de douane (suivi d'un vote postal) lors duquel plusieurs auditeurs affirmèrent se rappeler les Hungry Forties ${ }^{69}$.

Par ailleurs, un grand nombre d'autobiographies ouvrières très lues, influencées par le débat édouardien, furent rédigées en centrant les parcours individuels sur les années 1840, aussi marginale que cette décennie ait pu être en réalité pour les personnes concernées. Par exemple, un des principaux députés Lib-Lab qui exprimèrent les revendications des ouvriers ruraux avant 1914 (et après) était George Edwards, dont l'autobiographie From Crow-Scaring to Westminster fut publiée en $1922^{70}$. Le premier chapitre, intitulé "The Hungry Forties», décrivait la pauvreté vécue par ses parents dans le Norfolk rural, avant sa naissance en 1850. Dans une semblable veine urbaine, à Manchester, un réformateur social ruskinien, et fondateur de l'Ancoats Brotherhood en 1878, Charles Rowley (1839-1933), reconstitua lui aussi l'histoire de sa vie en commençant par les Hungry Forties : "nous avions abondance de détresse et de souffrance», à une époque où sa famille ne consommait de thé que durant les treize grossesses de sa mère (son père était gauchisseur et fabricant de cadres pour tableaux ${ }^{71}$. Parmi les exemples plus locaux figurent les souvenirs de James Ashworth de Blackburn, qui recréa sa vie de façon tout aussi stylisée ${ }^{72}$, tandis que toute une gamme d'autobiographies respectaient cette conceptualisation dans leurs réminiscences ${ }^{73}$.

En outre, les "Hungry Forties» imprégnèrent le discours universitaire aussi bien que l'histoire populaire entre les deux guerres. Par exemple, en 1919, dans ce qui constituait un résumé patriotique mais précis de la réussite économique britannique au XIX ${ }^{\mathrm{e}}$ siècle, William Page (1861-1934), plus connu comme responsable de la Victoria County History of England, notait sobrement : «Le pays était dans un état de détresse et de misère qui valut plus tard à cette période le nom sinistre de Hungry Forties " ${ }^{74}$. En 1923, une édition des mémoires de Robert Roberts (1834-1885) était présentée par son préfacier

69. The Listener, 2 avril 1930, p. 584; Ibidem (14 décembre 1932), C. R. Attlee. Voir aussi 'Bread: A Play for the Microphone', diffusé en juillet 1932, Radio Times, 460, 22 juillet 1932, p. 28. L'auteur de cette pièce radiophonique, Lawrence du Garde Peach, serait plus tard co-auteur du texte destiné au spectacle du centenaire de la Co-operative en 1944, voir plus haut, p. 57.

70. Londres, Labour Publishing Co.

71. Charles Rowley, Fifty years of Work without Wages (Laborare est Orare), Londres, Hodder \& Stoughton, s. d. (1911?), p. 3-10.

72. Blackburn Times, 3 mai 1924.

73. Dyke Wilkinson, Rough Roads; Reminiscences of a Wasted Life, Londres, Sampson Low, Marston \& Co. Ltd, 1912, p. 3 ; George R. Sims, évoquant le souvenir de son grand-père chartiste dans My life. Sixty Years' Recollections of Bohemian London, Londres, Eveleigh Nash, 1917, p. 8; Soutter, Fights for Freedom, p. 13-14, préface de John Burns, p. 33.

74. Commerce and Industry, 2 volumes, Londres, Constable, 1919, p. 127-128, 141-145, 147. Ce livre contenait une préface par sir William Ashley, économiste partisan de la réforme des droits de douane. Pour Page, voir http://www.history.ac.uk/makinghistory/resources/articles/william_page. html\#resources [consulté le 18 décembre 2015]. 
moderne comme "une description éloquente des conditions économiques des fermiers du pays de Galles dans les Hungry Forties " ${ }^{75}$. L'étude des migrations des travailleurs qu'Arthur Redford fit paraittre en 1926 incluait un chapitre intitulé "The Hungry Forties», mais il s'agissait d'un titre générique et non d'un point de départ pour son analyse ${ }^{76}$. Le concept informait encore des ouvrages historiques destinés à un lectorat plus populaire, comme The Common People de G. D. H. Cole et R.W. Postgate ${ }^{77}$ et le très influent English Social History de George Trevelyan ${ }^{78}$. La diffusion de l'expression est également soulignée par sa fréquence dans les manuels scolaires. Un échantillon de livres conçus pour différentes tranches d'âge indique qu'elle était encore couramment employée dans l'Entre-deux-guerres et dans l'immédiat aprèsguerre dans le secondaire, mais bien plus rarement dans les manuels pour les moins de 13 ans $^{79}$. Elle persista jusque dans les années 1950, même si on ne la rencontre plus dans les textes plus à jour, s'appuyant sur les recherches universitaires récentes ${ }^{80}$.

La formule Hungry Forties conserva une pertinence politique marquée, malgré son déclin : c'est un slogan qui fut régulièrement utilisé dans l'Entredeux-guerres, surtout dans le contexte de l'opposition libérale et travailliste à la politique économique des conservateurs. Par exemple, à la Chambre des Communes, le doyen du Parlement, T.P. O'Connor, s'exprimait en 1925 comme un vieil adepte du libre-échange : "Je veux protéger les masses contre le loup de la faim, que le libre-échange éloigna de leur porte durant les Hungry Forties ${ }^{81}$. La même année, le député travailliste Robert Richardson se rappelait également que, jeune homme, il avait connu un homme qui se rappelait «ne pas avoir eu de pain dans les Hungry Forties»; par conséquent, "Toute ma vie j'ai eu en horreur le mot même de protectionnisme " ${ }^{82}$. Quand le Gouvernement national introduisit en 1931-32 des mesures inspirées de celles de Joseph Chamberlain, ses opposants mentionnèrent à chaque fois les

75. John H. Davies (ed.), The Life and Opinions of Robert Roberts, wandering scholar, as told by himself, Cardiff, William Lewis, 1923.

76. Arthur Redford, Labour Migration in England, 1800-50, Manchester, The University Press, 1926, ch. VII, 118-131.

77. Londres, 1938, 1946.

78. Chapitre XXIV, 'The Hungry Forties', p. 291-309, réimpression de 1971, University Paperback; W.H. Chaloner, Hungry Forties, op. cit., 8-9; George M Trevelyan, English Social History, Harmondsworth, Penguin, 1944, p. 553, 558.

79. Cet exposé s'appuie sur un échantillon d'une trentaine de titres, tirés de la collection de manuels scolaires de l'Institute of Education de Londres. Parmi les exemples figurent Isidore Tenen, A History of England From the Earliest Times to 1932, Londres, Macmillan \& Co., 1932, p. 565-569; Anna Titterton, From Waterloo to George VI, Londres, Ginn, 1939, p. 57, 68-69; Edward J. S. Lay, The Modern Class-Book of English History. Senior Book V, Victoria and Modern Times, Londres, Macmillan \& Co, 1939, p. 27.

80. Il survécut ainsi dans la deuxième édition du livre de Donald G. Perry and Ronald M. S. Pasley, Modern British History, 1815-to 1939, Londres, A. \& C. Black, 1948, p. 491, mais pas dans Charles P. Hill, A Survey of British History, Book IV, 1783-1939, Londres, Edward Arnold, 1949, ouvrage influencé par les récents volumes de l'Oxford History of England. Hill resta un manuel standard tout au long des années 1950 et 1960 (expérience personnelle).

81. Hansard, 5 ${ }^{\mathrm{e}}$ série (185), c. 1781, 25 juin 1925.

82. Ibidem (183), c. 1269, 7 mai 1925. 
Hungry Forties, et le leader travailliste, Lansbury, prévoyait avec optimisme que «lorsqu'une fois encore, le peuple subira ce qu'il a enduré pendant les Hungry Forties", il se tournerait vers le socialisme ${ }^{83}$. De manière plus générale, alors que l'ombre de la faim planait sur les années 1930, le concept de Hungry Thirties fut façonné d'après le souvenir des années 1840, comme l'a montré James Vernon, et le message en fut étendu à une nouvelle génération politique : dans l'esprit du "Plus jamais ça», la démocratie sociale britannique garantissait désormais l'égalité de l'approvisionnement alimentaire puisque le rationnement sur le pain fut instauré en $1946^{84}$. La fréquence de l'expression après la guerre est bien reflétée par son choix, en 1949, comme titre de l'une des émissions de la BBC à destination des écoles évoquant «l'histoire du combat de Richard Cobden pour le pain bon marché durant les Hungry Forties» ${ }^{85}$.

Enfin, alors que 1946 marquait le centenaire de l'abrogation des Corn laws, la faim figurait à nouveau en bonne place dans le discours politique, non seulement national mais planétaire. À l'aube de l'ère de la gouvernance internationale, l'Organisation des Nations Unies pour l'alimentation et l'agriculture (FAO), tout juste créée, fut confrontée en 1946 au même problème de la faim, que Peel appelait en 1846 «le problème fondamental de la nourriture». Voyant les Hungry Forties comme une époque de misère intense à laquelle avait succédé une période de prospérité et de productivité, le service londonien des relations publiques de la FAO demandait : «Nos propres années 40, avec leur cortège de difficultés, finiront-elles de la même manière?» Mais, comme ceux qui percevaient les années 1840 comme l'apogée du mécontentement entre 1815 et $1846^{86}$, les hommes d'État après 1945 identifiaient aussi un problème à plus long terme, le quart-de-siècle précédent étant caractérisé dans l'histoire mondiale par la faim et la famine, et la FAO cherchait à établir le contrôle planétaire de l'alimentation et la "libération du besoin" comme base de la paix future ${ }^{87}$. Fait significatif, le premier directeur général de la FAO fut le déjà âgé Boyd Orr (1880-1971), qui avait pris conscience de la pauvreté et de la malnutrition dans les taudis

83. Hansard, (260), c. 2108,10 décembre 1931; (265), c. 688, 28 avril 1932, évoquant une de ses connaissances personnelles, un survivant des «Hungry Forties».

84. Par exemple, Fenner Brockway, Hungry England, Londres, Victor Gollancz, 1932; James Vernon, Hunger, op. cit., p. 236-71; Noel Annan, 'One hundred years ago', The Listener, 5 décembre 1946.

85. John Cotton Gagg, 'The Hungry Forties' (transcription d'une émission de la BBC), 2 juin 1949, W.E. Tate Papers, Museum of English Rural Life, MS 1234A/1/27; Tate, plus tard pionnier de l'histoire rurale et locale, était alors assistant de programmation ; BBC Written Archives, R16 Education general - Schools Programmes - History, 1938-1954; Radio Times, 1337, 27 mai 1949, p. 18; Manchester Guardian (3 mai 1949), p. 8. La Manchester Central Library organisa une exposition de livres en parallèle.

86. Entre autres, le dernier des «Cobdenites», Francis W. Hirst, The Repeal of the corn laws, with an account of the Hungry Forties, Midhurst, Cobden Club, 1946, p. 17-27.

87. The Hungry Forties (Public Relations Office for F.A.O. in the U.K., Londres, 1946). 
de Glasgow au début du $\mathrm{XX}^{\mathrm{e}}$ siècle, et qui devait faire allusion en connaissance de cause aux Hungry Forties, tant à la radio qu'à la chambre des Lords ${ }^{88}$.

\section{RÉVision ET TRANSMUtATION DEPUis LES ANNÉES I 950}

Cette extension planétaire du chrononyme Hungry Forties fut néanmoins le prélude de son effacement de la mémoire nationale, dans le cadre de la disparition plus générale des valeurs centrales de l'économie politique libérale du XIX ${ }^{\mathrm{e}}$ siècle et de ses épigones ${ }^{89}$. Déjà dans les années 1930, l'expression Hungry Forties avait été dénoncée comme "un mythe absurde» car les années 1840 «n'avaient pas été en Grande-Bretagne une période de pauvreté particulière ou de prix de l'alimentation spécialement élevés " ${ }^{90}$. La plupart des historiens de l'économie évitaient de l'employer. Sir John Clapham, premier professeur d'histoire économique à Cambridge, ne l'utilisa pas dans sa grande histoire de l'économie britannique et, dans une conférence donnée à Manchester en 1945, la rejeta explicitement, parce que les années 1840 n'étaient "pas plus affamées que les années 1830 ou 1850 »11. De même, T.S. Ashton, auteur d'une histoire de la Révolution industrielle devenue un classique, mais conférencier pour la Free Trade Union avant 1914, rejeta lui aussi la formule parce qu'elle «attachait le stigmate des Hungry Forties à la décennie qui vit l'essor des chemins de fer et l'abrogation des lois sur les grains $»^{92}$. Ce révisionnisme fut complet quand l'expression fut historicisée par un spécialiste d'histoire économique enseignant à Manchester, qui faisait autorité pour l'histoire du Nord-Ouest de l'Angleterre, épicentre des Hungry Forties dans la réalité comme dans la fiction. Dans sa brochure The Hungry Forties, Chaloner devint le premier historien à décrire clairement les origines édouardiennes de cet usage et sa transmission vers des ouvrages de référence canoniques comme l'édition 1910 (mais pas 1902) de l'Encyclopedia Britannica. De manière suggestive, Chaloner faisait remonter le déploiement édouardien de la formule à la "propagande de la faim entreprise par

88. Hansard, $5^{\mathrm{e}}$ série (178, Lords), c. 209, 23 juillet 1952, sur les prix alimentaires; J. Boyd Orr, 'The Road through Plenty to Peace', Listener, 1002, 17 juin 1948, d'après son allocution radiodiffusée, 10 juin, Radio Times, 1286, 4 juin 1948, p. 16.

89. Voir Frank Trentmann, 'The Resurrection and Decomposition of Cobden in Britain and the West', in Anthony Howe et Simon Morgan, (eds), Rethinking Nineteenth-Century Liberalism: Richard Cobden Bicentenary Essays, Aldershot, Ashgate, 2006, p. 264-288.

90. Montague Fordham, Britain's Trade and Agriculture: their recent evolution and future development, Londres, G. Allen \& Unwin Ltd, 1932, p. 28-29, cité par Chaloner, p. 10. Fordham, actif réformateur rural dans l'Angleterre édouardienne, connaissait manifestement les usages antérieurs de l'expression.

91. 'Corn Law Repeal, Free Trade and History', Transactions of the Manchester Statistical Society, Session 1945-1946, lu devant la Society, 10 octobre 1945; le Manchester Guardian n'apprécia que l'on abatte ainsi une "vache sacrée» locale, 11 octobre 1945, p. 4.

92. Thomas S. Ashton, 'The Treatment of Capitalism by Historians', in Friedrich A. Hayek (ed.), Capitalism and the Historians, Chicago, University of Chicago Press, p. 55. L'essai d'Ashton avait initialement été publié dans le Journal of Economic History, Supplement IX (1949). 
la Ligue pour l'abolition des lois sur les grains", mais son objectif principal était de détruire le mythe de la famine pendant l'essentiel de la décennie allant de 1840 à 1850, en s'appuyant sur les statistiques des salaires et de la consommation pour conclure : "Il ne reste donc pas grand-chose des Hungry Forties ${ }^{93}$. Cette rectification par Chaloner était aisément accessible car, après sa parution originale en 1957 comme premier volume de la série «Aids to Teachers" (aide aux enseignants) publiée par la Historical Association, elle connut six réimpressions dans les années 1960. Elle contribua à mettre un terme à l'usage populaire des Hungry Forties britanniques, d'autant que les conditions dans lesquelles ce débat s'était épanoui semblaient de plus en plus lointaines dans l'affluence de l'après-guerre.

Ce chrononyme malléable s’avéra pourtant durable de bien des façons. Par exemple, malgré l'apparition d'études historiques majeures sur la famine en Irlande, qui révélèrent le peu de pertinence de l'expression, les médias anglais l'appliquaient à un nombre croissant d'ouvrages littéraires et dramatiques très remarqués qui exploraient la "Grande Famine» irlandaise ${ }^{94}$. Ensuite, pour les historiens britanniques, cela restait une métaphore utile pour exprimer l'humeur du début des années 1840 comme période distinctive, le pire moment de détresse et de mécontentement populaire de tout le $\mathrm{XIX}^{\mathrm{e}}$ siècle, et donc source légitime de «mythes» ultérieurs. Enfin, comme indiqué plus haut, l'expression franchit la Manche, et pénétra le lexique de la nourriture et de la faim dans les origines des révolutions de 1848. Il est donc d'autant plus surprenant de constater que, jusque dans les années 1980, la formule était encore employée sans aucune distance critique, comme si elle avait été forgée dès les années 1840 . Même après de nouvelles réévaluations récentes du mythe des Hungry Forties, certains historiens répugnent à abandonner une expression dotée d'une telle puissance évocatrice, bien qu'anachronique.

Par bien des aspects, la formule Hungry Forties est donc comparable à ce que Hirsch appelle "post-mémoire", car la plupart des individus qui en usèrent et furent influencés par elle n'avaient pas directement vécu cette décennie mais appartenaient à une génération postérieure ${ }^{95}$. Malgré tout, sa

93. W.H. Chaloner, Hungry Forties, op. cit., p. 9. Chaloner n'incluait pas la Famine irlandaise dans sa vision des Hungry Forties.

94. Redcliffe N. Salaman, The History and Social Influence of the Potato, Cambridge, Cambridge University Press, 1949; R. Dudley Edwards et T. Desmond Williams (eds), The Great Famine: Studies in Irish History, 1845-52, Dublin, Browne \& Nolan, 1956; Mrs Cecil Woodham-Smith, The Great Hunger: Ireland, 1845-9, Londres, Hamish Hamilton, 1962; Times, 10 octobre 1956 (compte rendu de The Paddy Pedlar, de Michael Molloy; Times, 8 août 1961 (compte rendu d'une pièce radiophonique, $A$ grand year for mushrooms); George Cusack et Sarah Goss (eds), Hungry Words: Images of Famine in the Irish Canon, Dublin, Irish Academic Press, 2006.

95. Marianne Hirsch, The Generation of Postmemory: Writing and Visual Culture after the Holocaust, New York, Columbia University Press, 2012. 
construction dépendait des témoins vivants réunis dans The Hungry Forties de Jane Cobden Unwin, dont le «trauma» fut transmis et réinterprété par une génération plus tardive. Dans ce processus de médiation sociale et culturelle, des individus révisèrent l'histoire de leur propre vie et les Hungry Forties en vinrent à former une partie de leur identité. Mais pour beaucoup, la réalité des Hungry Forties devint bientôt insignifiante comparée à la peur de leur retour, angoisse politique suscitée dans le contexte politique spécifique de l'Angleterre du début du XX $\mathrm{XX}^{\mathrm{e}}$ siècle. Le récit dans lequel le protectionnisme et les taxes douanières compromettaient le bien-être populaire constitua pourtant par la suite un critère pour évaluer les mesures économiques et, dans les faits, les droits humains, la libération de la faim dans un contexte national. La post-mémoire créée à l'époque édouardienne n'était nullement dénuée d'importance future, d'autant que la faim apparut un siècle après au cour des préoccupations mondiales. Alors que la formule Hungry Forties souffrait dès son invention d'un manque d'exactitude, la mémoire qu'elle construisit, faite d'oppression et de rédemption, entra indubitablement dans la conscience nationale et n'en disparut que lentement, bien après que ses prétentions à la vraisemblance eurent été démenties. En tant que tel, un récit populaire avait été efficacement relayé comme histoire démocratique de la nation britannique, les souvenirs locaux et fragmentaires se transformant en mémoire populaire collective, avec ses propres images, motifs et récits autour $\mathrm{du} «$ Pain du Peuple». 